Paediatr Paedolog 2014 · [Suppl 1] 49:7-8 DOI 10.1007/s00608-014-0179-6

○) Springer-Verlag Wien 2014

\section{Thun-Hohenstein ${ }^{1,2}$}

${ }^{1}$ Universitätsklinik für Kinder- und Jugendpsychiatrie, Christian-Doppler-

Klinik, Paracelsus Medizinische Privatuniversität, Salzburg

${ }^{2}$ Politische Kindermedizin e. V., Wien

\title{
Partizipation in der Kinder- und Jugendmedizin
}

Partizipation ist eine sich durch alle Bereiche der Gesellschaft ziehende Grundhaltung der umfassenden Bürgerbeteiligung demokratischer Systeme, die ihren Ursprung in der Menschenrechtskonvention hat. Die Kinderrechtskonvention als Grundlage ethischen Handelns im Umgang mit Kindern und Jugendlichen und das Vordringen des partizipativen Gedankens in das Gesundheitswesen haben zu einer Veränderung des Umgangs mit kranken Kindern und Jugendlichen und ihren Angehörigen geführt.

Die vorliegende Ausgabe von pädiatrie \& pädologie fasst diese Entwicklungen in Europa zusammen und stellt Modelle für die Umsetzung des partizipativen Gedankens vor. Grundlage der Umsetzung sind Haltung und Strukturen, wie sie in der „child-friendly health care“ beschrieben und gefordert werden und in der EACH-Charta schon seit Jahrzehnten festgelegt sind.

Beispiele aus der Pädiatrie, dem Behindertenbereich, der Sozialpädiatrie und der Kinder- und Jugendpsychiatrie belegen die Notwendigkeit und Sinnhaftigkeit dieses Ansatzes, zeigen aber auch die Schwierigkeiten und Problemlagen der Umsetzung partizipativen Handelns im Gesundheitswesen auf.

\section{D) Partizipation muss eine Grundlage der medizinischen Ausbildung werden}

Partizipation gelingt nur dann, wenn die partizipative Grundhaltung als Grundlage der Ausbildung der verschiedenen Gesundheitsberufe gelehrt wird und die Ge- sundheitspolitik die Grundsätze des partizipativen Handelns in entsprechende Versorgungsstrukturen umsetzt. So hoffen wir, mit dieser Publikation die Diskussion um eine partizipative Haltung des Gesundheitswesen und insbesondere der Pädiatrie und Kinder- und Jugendpsychiatrie zu vertiefen und Anregungen zur Weiterentwicklung zu geben.

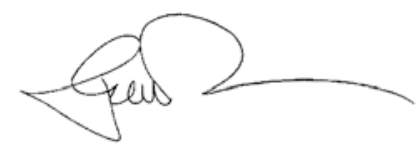

L. Thun-Hohenstein Salzburg, Juli 2014

Dieses Supplement ist das Ergebnis der 7. Jahrestagung der Politischen Kindermedizin in Salzburg 2013.

\section{Korrespondenzadresse}

Prof. Dr. L. Thun-Hohenstein Universitätsklinik für Kinder- und Jugendpsychiatrie, Christian-Doppler-Klinik, Paracelsus Medizinische Privatuniversität Ignaz-Harrer-Str. 79, 5020 Salzburg Österreich

I.thun-hohenstein@salk.at

Interessenkonflikt. L. Thun-Hohenstein gibt an, dass kein Interessenkonflikt besteht. 


\section{Hier steht eine Anzeige.}

算 Springer 